\title{
Effect of the Base of the Shoe Heel on Postural Stability During Walking in Women
}

\author{
Falola Jean-Marie ${ }^{1,2, ~ *}$, Koussihouèdé Fifamè Eudia Nadège ${ }^{1}$, Falola Stève Marjelin Donan ${ }^{1}$, \\ Avossevou Yves Gabriel ${ }^{3}$, Bio Nigan Issiako ${ }^{4}$, Dansou Houndjovi Pierre ${ }^{5}$ \\ ${ }^{1}$ Laboratory of Biomechanics and Performance (LABIOP), National Institute of Youth, Physical Education and Sport (INJEPS) University of \\ Abomey Calavi (UAC), Porto-Novo, Benin \\ ${ }^{2}$ Laboratory Human Motricity, Education, Sport, Health (LAMHESS) Training Unit and Research in Sciences and Techniques of Physical \\ and Sports Activities (STAPS) University of Nice Sophia Antipolis, Nice, France \\ ${ }^{3}$ Research Unit Theoretical Physics (URPT) Institute of Mathematics and Physical Sciences (IMSP) University of Abomey Calavi (UAC), \\ Porto-Novo, Benin \\ ${ }^{4}$ Laboratory APS and Motricity (LABAPSM), National Institute of Youth, Physical Education and Sport (INJEPS) University of Abomey- \\ Calavi (UAC), Porto-Novo, Benin \\ ${ }^{5}$ Laboratory of Exercise Physiology (LPE), National Institute of Youth, Physical Education and Sport (INJEPS) University of Abomey-Calavi \\ (UAC), Porto-Novo, Benin
}

\section{Email address:}

jemfalola@yahoo.fr (F. Jean-Marie),mjfalola@hotmail.com (F. Jean-Marie), eudiak@yahoo.fr (K. F. E. Nadège), famdos@hotmail.com (F. S. M. Donan), avossevou@yahoo.fr (A. Y. Gabriel), bionigan@yahoo.com (B. N. Issiako), kingpierre1@yahoo.fr (D. H. Pierre)

\section{To cite this article:}

Falola Jean-Marie, Koussihouèdé Fifamè Eudia Nadège, Falola Stève Marjelin Donan, Avossevou Yves Gabriel, Bio Nigan Issiako, Dansou Houndjovi Pierre. Effect of the Base of the Shoe Heel on Postural Stability During Walking in Women. American Journal of BioScience. Vol. 3, No. 5, 2015, pp. 167-170. doi: 10.11648/j.ajbio.20150305.11

\begin{abstract}
The high-heeled shoes are the cause of slips and falls while walking. Objective: To determine the shape of the base of the heel of the shoe to a stabilization of the locomotor pattern of walking. Materials and Methods: Fifteen young women had normal-weighted market shoes without heel and with heels of $5.08 \mathrm{~cm} ; 7.6 \mathrm{~cm}$ and $10 \mathrm{~cm}$. Each shoe were associated three square bases whose sides were: small: $1.5 \mathrm{~cm}$; average: $3 \mathrm{~cm}$ wide and $5.3 \mathrm{~cm}$. Results: Significant differences between the kinematic parameters were only for small and medium bases. There was no significant difference between the large base and the heel without shoes. The base of the heel of the shoe that stabilizes the locomotor pattern of walking is one that is large and whose side is equal to $5.3 \mathrm{~cm}$. This value represents the heel of the foot width of the subject.
\end{abstract}

Keywords: Gait, Kinematic Parameters, High Heels, Base of the Heel

\section{Introduction}

Human locomotion for over three million years, walking is a learned activity, integrated automatic (Plas and al., 1979). It is defined generally as a displacement consisting of a translation of the entire body, resulting from rotational movements of the joint (Bouisset and Maton, 2000). Walking is a cyclical activity characterized by a repeat sequences of body segments to move the body forwards while maintaining the balance (Perry, 1992). His decomposition involves two major phases that are the stance and swing that are evaluated through the space-temporal parameters.

By its nature, walking involves a near constant contact of the feet with the ground, which can cause injuries and other injuries to the sole of the foot. To remedy this state of affairs, the shoe was created for more than 5,500 years to protect the feet of different injuries they are subjected during walking (Pinhasi and al., 2010). Since then, the shoe has undergone several transformations to happen today, not only to play its historic main role, but also to serve as a fashion accessory, making all part of the clothing range. Thus the high-heeled shoes are now best worn by women in the performance of their daily and professional tasks.

This social behavior that tends to take more and more 
important, has interested many researchers. Thus, Hong and al. (2013), questioning on the stability factor, testified that women who wear high-heeled shoes often complain about the instability of the foot and lower back pain. Menz and Lord (1999) were interested in postural adaptation in high heels in the elderly people. They reveal that any changes occurring between the foot and the ground are likely to affect the posture. The authors concluded that high heels are sources of instability leading to injuries such as fractures and ankle sprains (Chien and al., 2013). Other studies had indicated that such a result by showing that the high heel disrupts the locomotor pattern and is associated with an increased risk of falling and slipping (Ebbeling and al., 1994).

Lord and Basford (1996) in a solution approach indicated that the increase of the base of the shoe heel part in the stability of the locomotor pattern. In addition, to improve postural stability in high-heeled shoes, the center of mass should be located 2 to $4 \mathrm{~mm}$, compared to the center line of the shoe (Philips and al., 1991). However, no details were given as to dimensions or measures that may have the base of the heel of the shoe to ensure stability of the locomotor pattern.

The objective of this study is to determine the shape of the base of the heel of the shoe for better stabilization of the locomotor pattern of walking.

\section{Materials and Methods}

\subsection{Type of Study and Implementation Framework}

This is a controlled prospective study, which took part in the Laboratory for Biomechanics and Performance (LABIOP) of the National Youth Institute of Physical Education and Sport (INJEPS)

\subsection{Study Population and Sampling}

The target population consists of women who had worn high-heeled shoes of public and private institutions of PortoNovo, one of the special status cities of Benin. The study was included a non-probability sample of fifteen healthy women with normal overweight $\left(\mathrm{BMI}=22.17 \pm 1.72 \mathrm{~kg} \mathrm{~m}^{-2}\right)$, volunteers, aged $22.4 \pm 2.56$ years and assigned the wearing high-heeled shoes as a professional dress requirement.

\subsection{Materials}

- Measurements of body mass and height were performed using respectively, scales (Seca, electronic, France) extended $150 \mathrm{~kg}$ and $0.1 \mathrm{~kg}$ precision and a fathom (206 Bodymeter Seca, Wall, France), range $2.20 \mathrm{~m}$, accurate to within $1 \mathrm{~mm}$.

- All the kinematic gait parameters were collected with OptoJump Next system (Microgate, version 1.8.10.0, Switzerland) which was connected to a computer where the data were transferred.

- The walk took place on a treadmill at a slope of $1 \%$ (Tehnogymn, slope $-5^{\circ}$ to $+25^{\circ}$, China) with one pair of shoes without heel and nine pairs of heels (Collection, wide base, Italy) as follows: Three heights of heels $5.08 \mathrm{~cm}, 7.62$ $\mathrm{cm}$, and $10 \mathrm{~cm}$. For each height were associated three different bases each having form of a square whose side was: small base $(\mathrm{Sb})=1.5 \mathrm{~cm}$; average basis $(\mathrm{Ab})=3 \mathrm{~cm}$; large base $(\mathrm{Lb})=5.3 \mathrm{~cm}$

- The heart rate at rest and walking were measured with a heart rate monitor (Polar RS 800 SD, Electronics, Finland).

- The step rate was imposed by a metronome (Seiko DM20, Digital, Deutschland) for the duration of walking on treadmill.

\subsection{Experimental Protocol}

A phase of addiction laboratory equipment was used to determine the speed gait freely chosen in shoes without heel $\left(\mathrm{S}_{\mathrm{fcwh}}\right)$. On this basis, the step frequency freely chosen in shoes without heel $\left(\mathrm{F}_{\text {fcwh }}\right)$ was also determined. The test consisted of walking on the treadmill at a slope of $+1 \%$, at the $\mathrm{S}_{\text {fcwh }}$ and $\mathrm{F}_{\text {fcwh }}$ for six minutes. Walking without shoes heel was the reference condition. In total, the subjects were walking in ten conditions that were all randomized. At the end of each passage, a recovery time is given to the subject to enable the return of heart rate to his the rest value.

\subsection{Variables Measured}

The kinematic parameters of the walking that are the stride length (SL), the proportion of the stance phase (PStP), the duration of the stance phase (DStP), the proportion of the swing phase (PSwP), the duration of the swing phase (DSwP) and the gear ratio (GR) were collected.

\subsection{Data Analysis}

The recorded data were processed using the Statistica software Stat Soft Inc (Version 7.1). For each variable the mean and standard deviation were calculated. The analysis of variance for repeated measures was used to determine generally the parameters affected by the shape of the base of the heel of the shoe. Fischer LSD test was used to determine significant differences specific to each shoe heel base. The level of statistical significance was set at $\mathrm{p}<0.05$.

\subsection{Ethical Considerations}

The study protocol was submitted to the Scientific Committee of Sectoral Sports and Physical Activities of Science and Technology of the University of Abomey-Calavi whose gave his approval to conduct the study before data collection. Each subject signed a card written informed consent before participating in the study.

\section{Results}

The characteristics of the subjects (Table 1) of this study showed that it was a young population with an average age of $22.40 \pm 2.56$ years. They said, considering the body mass index $(\mathrm{BMI}=22.17 \pm 1.72 \mathrm{~kg} \mathrm{~m}-2)$ that the study sample consists of normal-weighted women. The heel of the foot width of the subjects was $5.3 \mathrm{~cm}$.

The comparison of kinematic parameters at different shoe 
heels bases showed that whatever the height of the heel of the shoe, the large bead base had presented no significant difference. However, significant disturbances observed in the small and the average bead base increased with the height of the heel of the shoe. The bead base of the shoe that does not disturb the locomotor pattern is a square whose side is equal to the width of the heel of the foot of the subject.
Table 1. Biometrics subject characteristics.

\begin{tabular}{ll}
\hline & Mean \pm standard deviation \\
\cline { 2 - 2 } & Sample size $=\mathbf{1 5}$ \\
\hline Age (years) & $22.40 \pm 2.56$ \\
Height $(\mathrm{m})$ & $1.63 \pm 0.04$ \\
Body weight $(\mathrm{kg})$ & $59.07 \pm 5.15$ \\
Body mass index $\left(\mathrm{kg} / \mathrm{m}^{2}\right)$ & $22.17 \pm 1.72$ \\
Walking speed $(\mathrm{km} / \mathrm{h})$ & $2.63 \pm 0.17$ \\
Width of the heel of the foot $(\mathrm{cm})$ & $5.33 \pm 0.26$ \\
\hline
\end{tabular}

Table 2. Change in kinematic gait parameters at different shoe heels bases for a height of $5.08 \mathrm{~cm}$.

\begin{tabular}{lllllll}
\hline & SL (cm) & PStP (\%) & DStP(s) & PSwP (\%) & DSwP (s) & GR (m/pas/min) \\
\hline SWH & $61.3 \pm 8.05$ & $59.8 \pm 6.07$ & $0.803 \pm 0.265$ & $40.2 \pm 6.78$ & $0.531 \pm 0.113$ & $0.0058 \pm 0.0008$ \\
$\mathrm{Sb}$ & $54.75 \pm 9.38^{*}$ & $51.29 \pm 13.98^{*}$ & $0.623 \pm 0.185^{*}$ & $48.71 \pm 5.37 * * *$ & $0.594 \pm 0.153$ & $0.0051 \pm 0.0017$ \\
$\mathrm{Ab}$ & $61.75 \pm 6.75$ & $59.58 \pm 7.22$ & $0.842 \pm 0.211$ & $40.42 \pm 6.91$ & $0.580 \pm 0.186$ & $0.0052 \pm 0.007$ \\
$\mathrm{Lb}$ & $63.43 \pm 9.65$ & $59.63 \pm 6.91$ & $0.865 \pm 0.237$ & $40.37 \pm 7.22$ & $0.561 \pm 0.068$ & $0.0054 \pm 0.002$ \\
\hline
\end{tabular}

The numbers in the table represent the mean values \pm standard deviation of kinematics parameters; SL: step length; PStP: proportion of the stance phase; DStP: duration of the stance phase; PSwP: proportion of the swing phase; DSwP: duration of the swing phase; GR: gait ratio; *: Significant difference at $\mathrm{p}<0.05$; ***: Significant difference at $\mathrm{p}<0.001$

Table 3. Change in kinematic gait parameters at different shoe heels bases for a height of $7.6 \mathrm{~cm}$.

\begin{tabular}{lllllll}
\hline & SL $(\mathbf{c m})$ & PStP (\%) & DStP(s) & PSwP (\%) & DSwP(s) & GR (m/pas/min) \\
\hline $\mathrm{SWH}$ & $61.3 \pm 8.05$ & $59.8 \pm 6.07$ & $0.803 \pm 0.265$ & $40.2 \pm 6.78$ & $0.531 \pm 0.113$ & $0.0058 \pm 0.0008$ \\
$\mathrm{Sb}$ & $54.6 \pm 6.45^{*}$ & $50.8 \pm 3.5^{* * *}$ & $0.487 \pm 0.202^{* *}$ & $49.2 \pm 2.24 * * *$ & $0.571 \pm 0.152$ & $0.0327 \pm 0.0025^{* * *}$ \\
$\mathrm{Ab}$ & $60.25 \pm 7.53$ & $51.5 \pm 8.19^{* *}$ & $0.506 \pm 0.190^{* *}$ & $48.5 \pm 6.24^{* *}$ & $0.569 \pm 0.161$ & $0.0099 \pm 0.0026^{* * *}$ \\
$\mathrm{Lb}$ & $61.37 \pm 7.21$ & $61.1 \pm 7.3$ & $0.714 \pm 0.122$ & $38.9 \pm 6.62$ & $0.527 \pm 0.139$ & $0.0063 \pm 0.0049$ \\
\hline
\end{tabular}

The numbers in the table represent the mean values \pm standard deviation of kinematics parameters; SL: step length; PStP: proportion of the stance phase; DStP: duration of the stance phase; PSwP: proportion of the swing phase; DSwP: duration of the swing phase; GR: gait ratio; *: Significant difference at $\mathrm{p}<0.05$; **: Significant difference at $\mathrm{p}<0.01 ; * * *$ : Significant difference at $\mathrm{p}<0.001$

Table 4. Change in kinematic gait parameters at different shoe heels bases for a height of $10 \mathrm{~cm}$.

\begin{tabular}{llllll}
\hline & SL (cm) & PStP (\%) & DStP(s) & PSwP (\%) & DSwP (s) \\
\hline $\mathrm{SWH}$ & $61.3 \pm 8.05$ & $59.8 \pm 6.07$ & $0.803 \pm 0.265$ & $40.2 \pm 6.78$ & GR (m/pas/min) \\
$\mathrm{Sb}$ & $53.44 \pm 6.7 * *$ & $42.3 \pm 4.02 * * *$ & $0.382 \pm 0.336 * * *$ & $57.7 \pm 5.33 * * *$ & $0.531 \pm 0.113$ \\
$\mathrm{Ab}$ & $54.1 \pm 10.5 *$ & $51.5 \pm 2.5 * * *$ & $0.804 \pm 0.244$ & $48.5 \pm 5.34 * * *$ & $0.0058 \pm 0.0008$ \\
$\mathrm{Lb}$ & $55 \pm 10.4$ & $62.4 \pm 7.91$ & $0.835 \pm 0.261$ & $37.6 \pm 6.61$ & $0.566 \pm 0.075$ \\
\hline
\end{tabular}

The numbers in the table represent the mean values \pm standard deviation of kinematics parameters; SL: step length; PStP: proportion of the stance phase; DStP: duration of the stance phase; PSwP: proportion of the swing phase; DSwP: duration of the swing phase; GR: gait ratio; $*$ : Significant difference at $\mathrm{p}<0.05$; $* *$ : Significant difference at $\mathrm{p}<0.01 ; * * *$ : Significant difference at $\mathrm{p}<0.001$

\section{Discussion}

This study was conducted with a sample of women with high-heeled shoes. The normality of the locomotor pattern guided the choice of subjects for this study. Only women with a normal gait cycle have been taken into account. This was verified from the standards of literature behind than normal walking, the stance phase occupies $60 \%$ of the cycle and $40 \%$ swing phase (Viel, 2000; Vaughan and al. 1992) with a gear ratio which is invariant $0.0064 \mathrm{~m} / \mathrm{steps} / \mathrm{min}$ in women (Sekiya and al., 1997). Indeed, there was no significant difference between the values observed in subjects of this study and those of the literature.

This result confirms that of literature indicating that wearing high heels is associated with instability and collapse, which can lead to injuries such as rupture of the patellofemoral ligament (Hong and al., 2005). This could be due to the shape of the base of the heel of the shoe contacts the ground. Indeed, when the subject wear the shoe which has a small or medium base heel, the entire surface of its heel and the foot of the plant is not in contact with the ground; the support polygon which is the plane drawn by the footprints is thereby reduced, thus decreasing the balance strategies. The point of projection of the center of gravity and the center of pressure are then modified (Tortolero and al., 2007). And in such cases, while walking, the body's center of gravity could end up outside this polygon is restricted, leading to instability and imbalance; since in normal operation, the center of gravity moves to create a model of sine waves indicating the efficiency of walking, with correct durations of the various phases (Braune and Fischer, 1985). Walking in small or medium bases heels, disrupts this model ideal as reported in Stefanyshyn and al. (2000) who noticed that wearing high heels is a source of loss of fluidity of the market, hence the loss of efficiency. Indeed, they have indicated that a height of $4 \mathrm{~cm}$ heel, a significant difference at $\mathrm{p}<0.01$ was observed for the flight duration and therefore the swing phase. Which is in contradiction with the work of Ebbeling and al. (1994) did not observe significant differences that from $5.08 \mathrm{~cm}$ heel height. This discrepancy is justified by not taking into 
account the shape of the base of the heel in the course of their study. It is inferred that the results of studies by these authors did not specify the size of the bases used heeled shoes are in perspective because this study has shown that the base of the heel of the shoe strongly influences the quality of walking and that, whatever the height of the heel. Specifically, the small bead base absolutely influences walking heeled shoes, as well as how high.

Another important result of this study is the lack of significant difference between heel shoes without data and those observed with great shoe heel base. It is understood throughout this result that the large bead base with sides of the width of the heel of the foot, is one that offers a good balance about. What goes in the same direction as the results of Lord and Basford (1996) have shown that increasing the base of the shoe heel helps to improve postural stability. This was best explained by Phillips and al. (1991) who reported that the center of mass of the foot placed 2 to $4 \mathrm{~mm}$ with respect to the midline of the shoe, improving the postural stability during walking

\section{Conclusion}

Through the results of this study, the conclusion is that the determination of the base of the heel of the shoe that stabilizes the locomotor pattern is of paramount importance in the methodology to be used to study the effect of the heel height of the shoe on the foot. Studies on the effects of the height of the heel of the shoe on the locomotor pattern should take into account the base of the heel of the shoe to avoid methodological bias and collect real disturbances induced by high heels.

\section{Acknowledgements}

Acknowledgements to the Scientific Council of the University of Abomey which funded the TEAM POSPROFES for the realization of this study in the context of Interdisciplinary Research Projects and Inter Faculty of Competitive Grant Program for Research of the University of Abomey-Calavi ( $2^{\text {nd }}$ phase).

\section{Conflict of Interests}

The study received partial funding from the University of Abomey-Calavi.

\section{References}

[1] Bouisset S, Maton B. Muscles, postures et mouvements: base et applications de la méthode électromyographique. Paris: Hermann; 2000.

[2] Braune W, Fischer O. On the centre of gravity of the human body. Berlin: Springer Verlag; 1985.

[3] Chien HL, Liu MW, Lu TW, Kuo CC, Chung PC. Inter-joint sharing of total support moments in the lower extremities during gait in narrow-heeled shoes of different heights. J Biomech. 2013; 46(12): 2067-74.

[4] Ebbeling CJ, Hamill J, Crussemeyer JA. Lower extremity mechanics and energy cost of walking in high-heeled shoes. J Orthop Sports Phys Ther. 1994;19(4):190-6.

[5] Hong Y, Wang SJ, Lam WK, Cheung JT. Kinetics of Badminton Lunges in Four Directions. J Biomech. 2013; 46(12): 2067-74.

[6] Hong WH, Lee YH, Chen HC, Pei YC, Wu CY. Influence of heel height and shoe insert on comfort perception and biomechanical performance of young female adults during walking. Foot Ankle Int. 2005; 26(12):1042-8.

[7] Lord SR, Bashford GM. Shoe characteristics and balance in older women. J Am Geriatr Soc. 1996;44:429-33

[8] Menz HB, Lord SR. Footwear and postural stability in older people. J Am Podiatr Med Assoc. 1999;89: 346-357.

[9] Perry J. Gait Analysis, Normal and Pathological Function. Thorofare New Jersey: Slack; 1992. 48p.

[10] Phillips RD, Reczek DM, Fountain D, et al.. Modification of high-heeled shoes to decrease promotion during gait. J Am Podiatr Med Assoc. $1991 ;(81): 215$.

[11] Pinhasi R, Gasparian B, Areshian G, Zardaryan D, Smith A. First Direct Evidence of Chatolitic Footwear from the Near Eastern Highlands. PLoS ONE. 2010; 5(6).

[12] Plas F, Viel E, Blanc Y. La marche humaine. Kinésiologie dynamique, biomécanique et pathomécanique. 2èd. Paris: Masson; 1979.

[13] Sekiya N, Nagasaki H, Ito H, Furuna T. Optimal walking in terms of variability in step length. J Orthop Sports Phys Ther. 1997;26(5):266-72.

[14] Shefanyshyn DJ, Nigg BM, Fisher V, O'Flynn B, Liu W. The influence of high heeled hoes on kinematics, kinetics, and muscle EMG of normal female gait. J Appl Biomech. 2000; 16(3); 309-19.

[15] Tortolero X, Masani K, Maluly C, et al. Body Movement induced by Electrical Stimulation of Toe Muscles During Standing. Artif Organs. 2007; 32(1): 5-12.

[16] Vaughan CL, Davis BL, O'connor JC. Gait analysis laboratory. Champaign IL. Human kinetics Publishers. 1992;1-14.

[17] Viel E. La marche humaine, la course et le saut: biomécanique, explorations, normes et dysfonctionnements. Le Point en rééducation et en APS, 9. Paris : Masson ; 2009. ;267 p 